\title{
Efecto de la temperatura y concentración sobre las propiedades reológicas de la pulpa de mango variedad Tommy Atkins
}

\section{Effect of temperature and concentration on rheological properties of mango pulp variety Tommy Atkins}

\section{Efeito da temperatura e da concentração sobre as propriedades reológicas de polpa de manga variedade Tommy Atkins}

\author{
Fabián Alberto Ortega Quintana ${ }^{1 *}$; Eder Salcedo Galván²; Ronny Arrieta Rivero²; Ramiro Torres Gallo² \\ ${ }^{1}$ Facultad de Minas. Universidad Nacional de Colombia. Carrera 80 No 65-223-Núcleo Robledo. \\ Medellín, Colombia. \\ ${ }^{2}$ Facultad de Ingeniería. Universidad de Córdoba. Carrera 6 No 76-103. Montería, Colombia. \\ *faortegaqui@unal.edu.co; ingenierofabianortega@gmail.com
}

Fecha Recepción: 16 de febrero de 2015

Fecha Aceptación: 26 de junio de 2015

\begin{abstract}
Resumen
El mango Tommy Atkins es una de las variedades más explotadas en la industria y comercialización mundial. Es valorado por su larga vida útil y poca degradación en la manipulación y transporte. El rendimiento de la pulpa es alto, entre 60 y $75 \%$ del peso total del fruto, y es utilizada en la industria de refrescos, néctares, compotas y concentrados de pulpas. En este artículo se muestran los resultados del estudio del comportamiento y los parámetros reológicos de la pulpa concentrada de mango (Mangifera indica L.) variedad Tommy Atkins en el rango de temperatura $15-60^{\circ} \mathrm{C}$ y en el rango de concentración 15-30 Brix. Para esto, fue utilizado un viscosímetro Brookfield Modelo DV-II+Pro (R). La pulpa de mango presentó un comportamiento no Newtoniano seudoplástico a todas las temperaturas y concentraciones evaluadas. La relación entre el esfuerzo de corte y la velocidad de cizalla fue modelada por la Ley de Potencia o modelo de Ostwald de Waele. Los modelos de Arrhenius y la ecuación de potencia fueron ajustados adecuadamente a los datos de la viscosidad aparente de la pulpa con respecto a la temperatura y la concentración. Los valores encontrados permitirán mejorar el diseño de equipos de transporte y tratamiento térmico de la pulpa del mango Tommy Atkins.
\end{abstract}

Palabras clave: reología, viscosidad aparente, seudoplástico, índice de consistencia.

\begin{abstract}
Tommy Atkins mango is one of the varieties most exploited in the industry and marketing global. It is valued for its shelf life and low degradation in handling and transport. The pulp yield is high, between 60 and $75 \%$ of the total weight of the fruit, and it is used in the soft drink industry, nectars, compotes and pulp concentrates. This paper shows the behavior and rheological parameters of concentrated mango pulp to different temperatures and concentrations using a Brookfield Modelo DV-II+Pro (R) viscometer. The mango pulp exhibited non-Newtonian pseudoplastic behavior to the temperatures and concentrations studied. The relationship between the shear stress and shear rate was modeled by Power Law or Ostwald de Waele model. Variations in apparent viscosity with respect to temperature and concentration were modeled with the Arrhenius model and power equation, respectively. The results will improve the design of transport equipment and thermal treatment of mango pulp variety Tommy Atkins.
\end{abstract}

Keywords: rheology, apparent viscosity, pseudoplastic, index of consistency. 


\section{Resumo}

Manga Tommy Atkins é uma das variedades mais exploradas na indústria e marketing global. É apreciada pela sua longa vida e baixa degradação no manuseio e transporte. O rendimento da polpa é elevada, entre 60 e $75 \%$ do peso total do fruto, e é utilizado na indústria de refrescos, néctares, compotas e polpa concentrada. Neste artigo de investigação, os resultados do estudo de comportamento e parâmetros reológicos de polpa de manga concentrado (Mangifera indica L.) Tommy Atkins, na gama de temperaturas de $15-60^{\circ} \mathrm{C}$ e na gama de concentração de $15-30^{\circ}$ Brix, são mostrados. Para isso, foi utilizado um viscosímetro Brookfield modelo DV-II + Pro (R). Polpa de manga apresentou um comportamento pseudoplástico não-newtoniano em todas as temperaturas e concentrações. A relação entre a tensão de corte e velocidad de cizallae foi modelado pelo modelo de lei de Potência ou de Ostwald de Waele. O Modelo de Arrhenius e equação de Potência foram devidamente ajustados aos dados experimentais da viscosidade aparente da polpa em relação à temperatura e concentração. Os valores vão melhorar a concepção de equipamentos de transporte e tratamento térmico de polpa de manga Tommy Atkins.

Palabras-chave: reologia, viscosidade aparente, seudoplástica, índice de consistência.

\section{Introducción}

Los productos obtenidos a base de las frutas, tales como jugos, néctares, mermeladas, pastas, salsas y helados contienen las pulpas como materias primas básicas. Estas pulpas pueden ser transportadas a través de tuberías, agitadas y mezcladas en tanques con otras materias primas, pasteurizadas y evaporadas en intercambiadores de calor y evaporadores continuos. Para que estas operaciones unitarias sean técnica y económicamente factibles es importante tener el conocimiento de las propiedades de la pulpa. Entre estas propiedades una de las más importantes es el comportamiento reológico, siendo usado como medida de la calidad y en la evaluación y operación de los equipos de procesamiento [1].

Las medidas reológicas han sido consideradas como una herramienta analítica que arroja información de la organización estructural de los alimentos. Varios factores afectan el comportamiento reológico de las pulpas de frutas que incluyen la temperatura [2-3], concentración de sólidos solubles totales [4] y el tamaño de partícula [5].

Como resultado de la interacción compleja entre los azúcares, las sustancias pécticas y los sólidos en suspensión, la gran mayoría de las pulpas de frutas son fluidos no Newtonianos [6-7]. Diferentes ecuaciones han sido usadas para la descripción reológica de productos líquidos a base de frutas: Modelo de Bingham, modelo de Ostwald de Waele o Ley de Potencia, modelo de Herschel y Bulkley, modelo de Casson, modelo de MizrahiBerk, entre otros [8]. Muchos alimentos líquidos tienen un comportamiento reológico que puede ser caracterizado usando el modelo de Ley de Potencia o de Ostwald de Waele [9-10], ya que se ajusta bien con los datos experimentales, siendo un modelo simple y con amplia aplicación tecnológica [11-12].

$$
\sigma=K \dot{\gamma}^{n}
$$

donde $\sigma$ es el esfuerzo de corte $(\mathrm{Pa}), \dot{\gamma}$ es la velocidad de cizalla $\left(\mathrm{s}^{-1}\right), K$ es el índice de consistencia $\left(\mathrm{Pa} . \mathrm{s}^{n}\right)$ y $n$ es el índice de comportamiento de flujo (adimensional).

Además, una ecuación tipo Arrhenius se puede utilizar para describir la influencia de la temperatura sobre la viscosidad aparente $\left(\eta_{a p p}\right)$ a una velocidad de cizalla constante [9]:

$$
\eta_{a p p}=\eta_{0} e^{\frac{E_{a}}{R T}}
$$

Donde $T$ es la temperatura absoluta, $R$ : la constante universal de los gases, $\eta_{0}$ : el factor preexponencial y $E_{a}$ : la energía de activación para la viscosidad

La ecuación de Arrhenius también es usada para describir la influencia de la temperatura sobre el índice de consistencia $K$.

$$
K=K_{0} e^{\frac{E a}{R T}}
$$

Donde $K_{0}$ es el factor pre-exponencial.

Por otro lado, las variaciones de la viscosidad aparente y del índice de consistencia con la concentración pueden ser descritas por una ecuación de potencia. 


$$
\begin{gathered}
K=a C^{b} \\
\eta_{a p p}=\eta_{1} C^{\alpha}
\end{gathered}
$$

Donde $C$ es la concentración de la pulpa en ${ }^{\circ}$ Brix $, a, b, \eta_{1}$ y $\alpha$ son parámetros de cada modelo respectivo.

El efecto de la temperatura y de la concentración sobre las variaciones del índice de consistencia y de la viscosidad aparente pueden ser descritas por las Ecuaciones 6 - 8.

$$
\begin{aligned}
& K=K_{1} C^{n_{1}} e^{\frac{E a}{R T}} \\
& \left.K=K_{2} e^{\left(\frac{E a}{R T}+n_{2} C\right.}\right) \\
& \eta_{\text {app }}=\delta C^{\varepsilon} e^{\frac{E a}{R T}}
\end{aligned}
$$

Donde $K_{1}, n_{1}, K_{2}, n_{2}, \delta$ y $\varepsilon$ son parámetros de cada modelo, respectivamente.

El efecto combinado de la temperatura, concentración y la velocidad de cizalla sobre la variación de la viscosidad aparente puede ser descrito por la Ecuación 9.

$$
\eta_{a p p}=K_{T, \dot{\gamma}, C} \dot{\gamma}^{\bar{n}-1} e^{\left[\left(\frac{E a}{R T}\right)+B C\right]}
$$

Donde $K_{T, \hat{\gamma}, C}$ y $B$ son parámetros ajustables del modelo y $\bar{n}$ es el promedio de todos los valores del índice de comportamiento de flujo [13].

El mango es conocido como el "rey de las frutas" debido a su aroma pronunciado y a su sabor distintivo. Existen numerosas variedades de mango que difieren entre sí por la forma, la piel, el color y las características de la pulpa. El mango Tommy Atkins es una de las variedades más explotadas en la industria y comercialización mundial. Es valorado por su larga vida útil y poca degradación en la manipulación y transporte. El rendimiento de la pulpa es alto, entre 60 y $75 \%$ del peso total del fruto, y es utilizada en la industria de refrescos, néctares, compotas y concentrados de pulpas. Las propiedades reológicas de las pulpas de mango dependen mucho de sus variedades, estado de maduración, concentración de sólidos solubles y temperatura [14]. Sin embargo, los valores típicos del índice de comportamiento de flujo, del índice de consistencia y de la energía de activación de flujo son bastante limitados en la literatura, considerando la alta disponibilidad de variedades de mangos. Por lo tanto, esta investigación fue realizada para determinar el comportamiento y los parámetros reológicos de la pulpa concentrada de mango (Mangifera indica L.) variedad Tommy Atkins en el rango de temperatura $15-60^{\circ} \mathrm{C}$ y en el rango de concentración $15-30^{\circ}$ Brix.

\section{Parte experimental}

\section{Materia prima}

Las pulpas fueron obtenidas de mangos variedad Tommy Atkins seleccionados, lavados, escaldados a $95^{\circ} \mathrm{C}$ por $5 \mathrm{~min}$, pelados, despulpados con una malla de diámetro $1,5 \mathrm{~mm}$ y concentradas en un evaporador rotatorio Heidolph ${ }^{\circledR}$. La medida de las concentraciones fue realizada mediante un refractómetro Bertuchi ${ }^{\circledR}$ a $25^{\circ} \mathrm{C}$ según Método AOAC. 981.12 de 1990. Finalmente, la pulpa concentrada fue empacada en bolsas herméticas y refrigerada a $4^{\circ} \mathrm{C}$ hasta 4 horas antes de la realización de los análisis reológicos. Se realizó una caracterización fisicoquímica de la pulpa determinando su acidez y $\mathrm{pH}$ por los métodos oficiales de la AOAC 942.15/90 y 932.12/90, respectivamente [15].

\section{Medidas reológicas}

Las medidas reológicas se realizaron en pulpas con concentraciones de sólidos solubles de 15 , 20, 25 y $30^{\circ}$ Brix, temperaturas de la pulpa 15, 30, $45,60^{\circ} \mathrm{C}$ y velocidades de cizalla 0,$93 ; 1,86 ; 2,79$; 4,$65 ; 9,3 ; 18,6 ; 27,9 ; 37,2 ; 46,5 ; 55,8 ; 65,1 ; 74,4$; 83,$7 ; 93 \mathrm{~s}^{-1}$. Estas temperaturas y concentraciones corresponden al rango normalmente utilizado en la industria procesadora de frutas.

A las muestras de pulpas de $5 \mathrm{~cm}^{3}$ se realizaron rampas de velocidad con cilindros concéntricos en viscosímetro Brookfield Model DV-II+Pro ${ }^{\circledR}$ utilizando un splinder S-61. Se varió la velocidad de cizalla de 0 a $93 \mathrm{~s}^{-1}$ en forma ascendente $y$ descendente con un tiempo de espera de 10s. Esta prueba se realizó para determinar la existencia o no de tixotropía.

\section{Ajuste a los modelos reológicos}

Los datos experimentales de la pulpa concentrada de mango fueron ajustados a los modelos reológicos de Ley de Potencia, Bingham, Casson y Herschel-Bulkey. Se utilizó el software Statgraphics Centurion $X V$ y se tomaron como criterios estadísticos la suma del cuadrado del error (SCE) y 
el coeficiente de determinación $\left(r^{2}\right)$. La tixotropía fue evaluada por pruebas de comparación de medias $(p<0,05)$ para la variable de respuesta esfuerzo de corte a una velocidad de cizalla $\left(0-93 s^{-1}\right)$ en forma ascendente y descendente.

También se ajustaron los datos experimentales de la pulpa concentrada de mango a los modelos expresados por las Ecuaciones 3, 4, 6 y 7, para modelar el índice de consistencia frente a la temperatura, frente a la concentración y frente a los efectos combinados de temperatura y concentración respectivamente; al igual que a los modelos expresados por las Ecuaciones 2, 5, 8 y 9 para modelar la viscosidad aparente frente a la temperatura, concentración, los efectos combinados de temperatura y concentración y frente a los efectos combinados de temperatura, concentración y gradiente de concentración, respectivamente.

Por último, se utilizó una curva maestra para interpretar los reogramas de la pulpa de mango concentrada a las diferentes temperaturas y concentraciones. Un total de 16 reogramas fueron combinados usando la técnica de superposición para formar una curva maestra con doble corrección por desplazamiento. Para la primera corrección, se seleccionó la temperatura de referencia y un esfuerzo de corte base teniendo en cuenta la mejor representación gráfica. Para cada concentración, las demás temperaturas fueron desplazadas horizontalmente a lo largo del eje de velocidad de cizalla a la temperatura de referencia para obtener los factores adimensionales de desplazamiento o corrección de velocidad de cizalla por temperatura $\left(a_{T}\right)$, definidos como se muestra en la Ecuación 10.

$$
a_{T, i}=\frac{\dot{\gamma}_{i}}{\dot{\gamma}_{R}}
$$

donde $\dot{\gamma}_{R}$ es la velocidad de cizalla a la temperatura referencia, $\dot{\gamma}_{i}$ es la velocidad de cizalla a otra temperatura evaluada a esfuerzo de corte base (10Pa) y $\dot{\gamma} / a_{T}$ es llamado primera velocidad de cizalla reducida $\left(\dot{\gamma}^{\prime}\right)$.

La curva maestra fue graficada teniendo como ejes el esfuerzo de corte y la velocidad de cizalla dividido por el factor de corrección por temperatura $\left(\dot{\gamma} / a_{T}\right)$. El desplazamiento horizontal con $a_{T}$ combina las cuatro temperaturas en una curva maestra para cada concentración. La ecuación de la Ley de Potencia (Ecuación 11) fue ajustada para las curvas maestras de las cuatro concentraciones para obtener la expresión del comportamiento reológico de las pulpas en términos del índice de consistencia $\left(K_{l}\right)$ y el índice de comportamiento de flujo $\left(n^{\prime}\right)$.

$$
\sigma=K^{\prime}\left(\frac{\dot{\gamma}}{a_{T}}\right)^{n^{\prime}}
$$

A las cuatro curvas maestras de concentración se les realizó una segunda corrección, utilizando los mismos criterios de la primera, pero ahora tomando como referencia la concentración y un esfuerzo de corte base para construir una curva maestra única usando el factor de corrección de concentración $a_{C}$ de acuerdo con la Ecuación 10, donde la segunda velocidad de cizalla reducida, $\left(\dot{\gamma}^{\prime \prime}\right)$, es cuantificado como $\dot{\gamma} / a_{T} / a_{c}$. La curva maestra final fue graficada como esfuerzo de corte frente a la velocidad de cizalla divido por los factores de corrección de temperatura $\left(a_{T}\right)$ y de corrección de concentración $\left(a_{c}\right)$ para estimar el comportamiento reológico de la pulpa a las concentraciones de $15-30^{\circ}$ Brix y temperatura de $15-60^{\circ} \mathrm{C}$. La curva maestra fue también ajustada a la ecuación de Ley de Potencia (Ecuación 12) para obtener una expresión única del comportamiento reológico de la pulpa en términos del índice de consistencia $\left(K^{\prime \prime}\right)$ y el índice de comportamiento de flujo $\left(n^{\prime \prime}\right)$.

$$
\sigma=K^{\prime \prime}\left(\frac{\dot{\gamma}}{a_{T} a_{C}}\right)^{n^{\prime \prime}}
$$

\section{Resultados y Discusión}

\section{Análisis fisicoquímicos:}

En la Tabla 1 se muestran los análisis fisicoquímicos realizados a la pulpa de mango Tommy Atkins.

Tabla 1. Análisis fisicoquímico de la pulpa de mango.

\begin{tabular}{ccc}
\hline Análisis & Método & Resultado \\
\hline Acidez [\%p] & $942.15 / 90 \mathrm{AOAC}$ & $0,283 \pm 0,09$ \\
$\begin{array}{c}\text { Sólidos solubles } \\
\left.\text { totales [ }{ }^{\circ} \mathrm{Brix}\right]\end{array}$ & $981.12 / 90 \mathrm{AOAC}$ & $16,4 \pm 0,17$ \\
$\mathrm{pH}$ & $932.12 / 90 \mathrm{AOAC}$ & $4,37 \pm 0,03$ \\
\hline
\end{tabular}

Comportamiento reológico de la pulpa de mango

En la Figura 1 se muestran las curvas de flujo típicas de la pulpa para la concentración de $30^{\circ} \mathrm{Brix}$, las concentraciones 15,20 y $25^{\circ}$ Brix tuvieron una variación similar del esfuerzo de corte frente a la velocidad de cizalla. No hubo tixotropía entre los datos experimentales con velocidad de cizalla ascendente $\left(0-93 s^{-1}\right)$ y con velocidad de 
cizalla descendente $\left(93-0 \mathrm{~s}^{-1}\right)$, por lo tanto, los valores mostrados son los valores promedios. Estos resultados están de acuerdo con Ramos AM e Ibarz A. [16], quienes expresaron que la tixotropía es comúnmente exhibida en puré y jugos de frutas concentrados con sólidos solubles totales mayores de $55^{\circ}$ Brix. Ninguna de las muestras de pulpa requirió de un esfuerzo de corte inicial $\left(\sigma_{0}\right)$, lo que sugiere que la pulpa de mango no posee una estructura ramificada fuerte que necesita de una fuerza para su ruptura antes que el flujo pueda ocurrir [17].

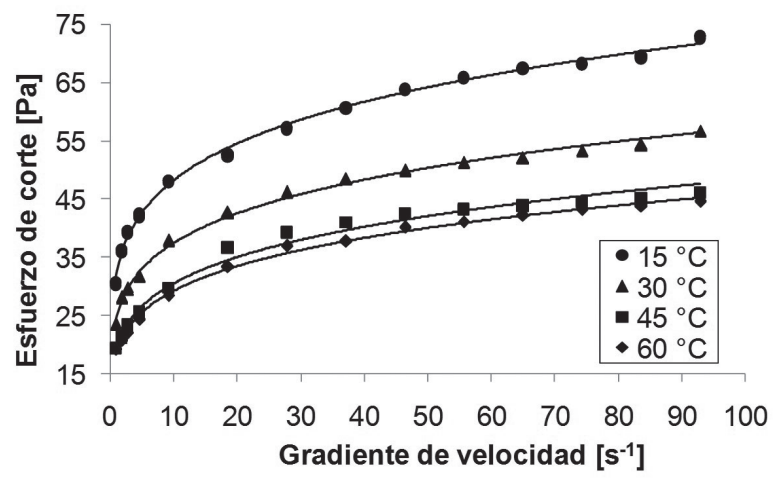

Figura 1. Reogramas de la pulpa a $30^{\circ}$ Brix a diferentes temperaturas.

El modelo de Ostwald de Waele $\left(0,957<r^{2}<0,997\right.$ y $28,87<$ SCE $<29,53$ ) se ajustó mejor a los datos experimentales que los modelos de Bingham $\left(0,817<r^{2}<0,905\right.$ y $\left.65,21<\mathrm{SCE}<73,91\right)$, Casson $\left(0,854<r^{2}<0,938\right.$ y $58,91<$ SCE $\left.<64,53\right)$ y HerschelBulkey $\left(0,927<r^{2}<0,962\right.$ y $48,83<$ SCE $\left.<52,51\right)$.
Como muestra la Tabla 2, la pulpa de mango exhibió un comportamiento reológico de un fluido no Newtoniano seudoplástico debido a que los valores del índice de comportamiento de flujo $(n)$ fue menor que $1(n<1)$ para todas las temperaturas y concentraciones.

Tabla 2. Índice de comportamiento de flujo $(n)$ e índice de consistencia $\left(K: P a . s^{n}\right)$ de la pulpa a diferentes temperaturas.

\begin{tabular}{|c|c|c|c|c|c|}
\hline \multirow{2}{*}{$\begin{array}{c}\text { Temperatura } \\
{\left[{ }^{\circ} \mathrm{C}\right]}\end{array}$} & \multirow{2}{*}{ Parámetro } & \multicolumn{4}{|c|}{ Concentración [ [Brix] } \\
\hline & & 15 & 20 & 25 & 30 \\
\hline \multirow{3}{*}{15} & K & $6,0221 \pm 0,165$ & $7,8551 \pm 0,133$ & $21,7412 \pm 0,182$ & $31,9049 \pm 0,187$ \\
\hline & $n$ & $0,1926 \pm 0,007$ & $0,2581 \pm 0,004$ & $0,2280 \pm 0,002$ & $0,1782 \pm 0,002$ \\
\hline & $r^{2}$ & 0,957 & 0,993 & 0,997 & 0,997 \\
\hline \multirow{3}{*}{30} & $K$ & $5,4644 \pm 0,094$ & $6,7210 \pm 0,110$ & $17,3366 \pm 0,202$ & $24,7445 \pm 0,171$ \\
\hline & $n$ & $0,2021 \pm 0,004$ & $0,2409 \pm 0,004$ & $0,2269 \pm 0,003$ & $0,1812 \pm 0,002$ \\
\hline & $r^{2}$ & 0,986 & 0,991 & 0,993 & 0,996 \\
\hline \multirow{3}{*}{45} & $K$ & $4,2253 \pm 0,065$ & $6,1803 \pm 0,159$ & $12,0987 \pm 0,299$ & $19,6407 \pm 0,266$ \\
\hline & $n$ & $0,2181 \pm 0,004$ & $0,2372 \pm 0,006$ & $0,2592 \pm 0,006$ & $0,1937 \pm 0,004$ \\
\hline & $r^{2}$ & 0,994 & 0,984 & 0,980 & 0,986 \\
\hline \multirow{3}{*}{60} & $K$ & $4,0302 \pm 0,109$ & $5,2633 \pm 0,134$ & $10,5555 \pm 0,163$ & $18,5946 \pm 0,143$ \\
\hline & $n$ & $0,2168 \pm 0,006$ & $0,2547 \pm 0,006$ & $0,2752 \pm 0,004$ & $0,1961 \pm 0,002$ \\
\hline & $r^{2}$ & 0,985 & 0,982 & 0,993 & 0,996 \\
\hline
\end{tabular}


Índice de comportamiento de flujo e índice de consistencia

En este estudio, considerando el modelo de Ostwald de Waele, los valores de $n$ variaron desde 0,1812 a 0,2752 y el índice de consistencia $(K)$ varió desde 4,0302 a 31,9049Pa. ${ }^{\mathrm{n}}$. Resultados similares fueron obtenidos en jugo de mango Kesar $(0,2<n<0,33$ y $\left.0,32<K<80,09 \mathrm{~Pa} . \mathrm{s}^{\mathrm{n}}\right)$ por Dak M et al. [14], en pulpa de mango $\left(0,25<n<0,78\right.$ y $\left.1<K<50 \mathrm{~Pa} . \mathrm{s}^{\mathrm{n}}\right)$ por Krokida
$\mathrm{M}$ et al. [18] y en pulpa de mango Chausa $(0,2<n$ $<0,9$ y $3<K<40 \mathrm{~Pa} . \mathrm{s}^{\mathrm{n}}$ ) por Khandari $\mathrm{P}$ et al. [19].

La Figura 2 muestra el comportamiento del índice de consistencia frente al inverso de la temperatura absoluta para las diferentes concentraciones de pulpa de mango. La Ecuación 3 fue ajustada a los datos de índice de consistencia e inverso de temperatura, cuyos parámetros se visualizan en la Tabla 3.

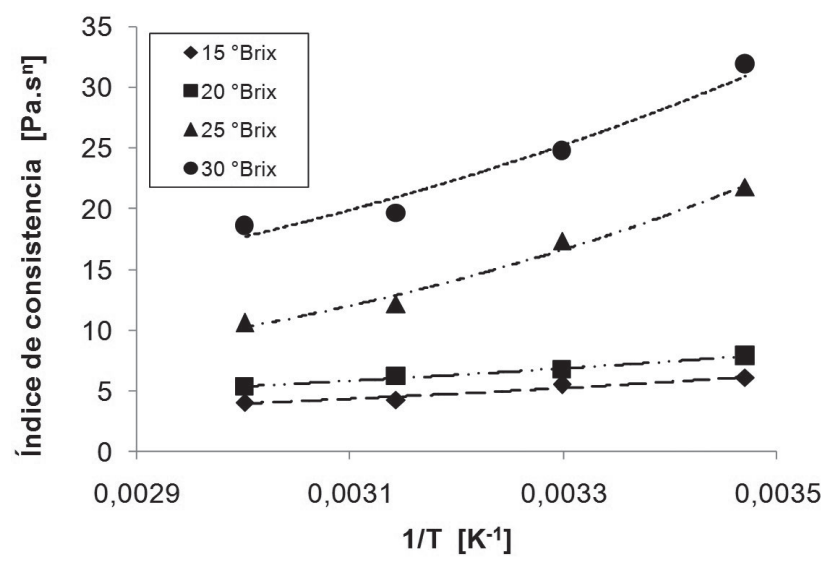

Figura 2. Índice de consistencia frente al inverso de temperatura absoluta a diferentes concentraciones de la pulpa.

Tabla 3. Energía de activación de flujo de la pulpa de mango.

\begin{tabular}{|c|c|c|c|}
\hline $\begin{array}{c}\text { Concentración } \\
{\left[{ }^{\circ} \text { Brix }\right]}\end{array}$ & $\begin{array}{c}K_{0} \\
{\left[\mathrm{~Pa} . \mathrm{s}^{\mathrm{n}}\right]}\end{array}$ & $\begin{array}{l}\text { Energía de activación (Ea) } \\
{[\mathrm{kJ} / \mathrm{mol}]}\end{array}$ & $r^{2}$ \\
\hline 15 & $0,2392 \pm 0,061$ & $7,76 \pm 0,65$ & 0,950 \\
\hline 20 & $0,4647 \pm 0,053$ & $6,77 \pm 0,25$ & 0,986 \\
\hline 25 & $0,0794 \pm 0,030$ & $13,47 \pm 0,63$ & 0,983 \\
\hline 30 & $0,3909 \pm 0,020$ & $10,51 \pm 0,56$ & 0,971 \\
\hline
\end{tabular}

Se observa una correlación positiva entre el índice de consistencia $(K)$ y el inverso de la temperatura absoluta $(1 / T)$ para todas las concentraciones. La energía de activación de flujo varió desde 7,76 a $13,47 \mathrm{~kJ} / \mathrm{mol}$, lo cual está de acuerdo con los valores reportados para alimentos líquidos y semilíquidos [20].

La Figura 3 muestra el índice de consistencia frente a la concentración. La Ecuación 4 fue ajustada a los datos de índice de consistencia y concentración, cuyos parámetros se visualizan en la Tabla 4. Se observa que el índice de consistencia incrementa de manera no lineal con el incremento en la concentración a una temperatura específica.
Estos resultados son consistentes con estudios reportados para otras frutas (tomate, piña, naranja y mango variedad Chausa) [19,21-23].

El efecto combinado de la temperatura y concentración sobre el índice de consistencia de la pulpa de mango fue predicho por las Ecuaciones 6 y 7 , cuyos parámetros se muestran en la Tabla 5. La energía de activación $\left(E_{a}\right)$, para ambas ecuaciones, arroja valores similares y ambas ecuaciones sugieren buen ajuste a los datos experimentales obtenidos. Estos resultados estuvieron de acuerdo con diferentes investigaciones sobre jugos concentrados de uva, de cereza, de granada y de guanábana [24-27]. 


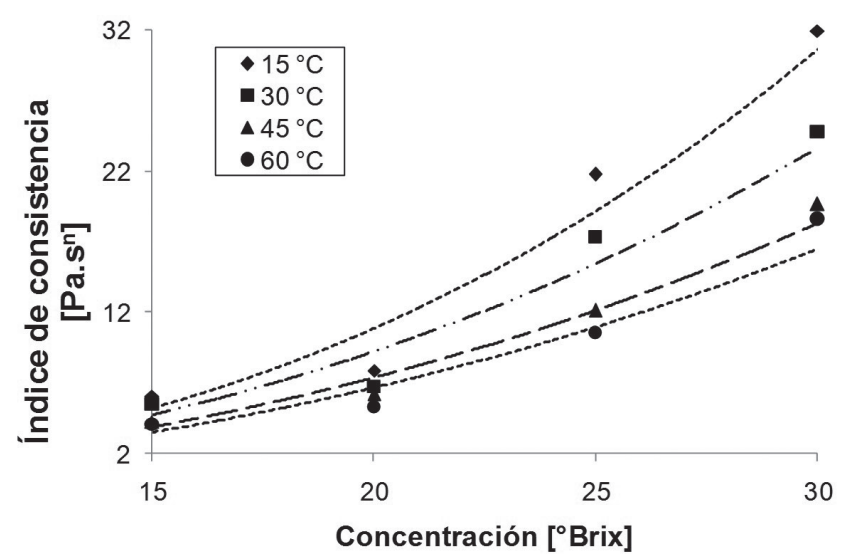

Figura 3. Índice de consistencia frente a la concentración a diferentes temperaturas de la pulpa.

Tabla 4. Parámetros del índice de consistencia ajustado a la Ecuación 4.

\begin{tabular}{cccc}
\hline Temperatura $\left[{ }^{\circ} \mathbf{C}\right]$ & $\boldsymbol{a}$ & $\boldsymbol{b}$ & $\boldsymbol{r}^{2}$ \\
\hline 15 & $0,0027 \pm 0,004$ & $2,7651 \pm 0,20$ & 0,972 \\
30 & $0,0043 \pm 0,003$ & $2,5535 \pm 0,21$ & 0,967 \\
45 & $0,0031 \pm 0,002$ & $2,5719 \pm 0,11$ & 0,991 \\
60 & $0,0014 \pm 0,001$ & $2,7822 \pm 0,17$ & 0,981 \\
\hline
\end{tabular}

Tabla 5. Parámetros del índice de consistencia ajustado a las Ecuaciones 6 y 7.

\begin{tabular}{lll}
\hline \multicolumn{1}{c}{ Modelo ajustado } & \multicolumn{1}{c}{$K=K_{1} C^{n_{1}} e^{\frac{E a}{R T}}$} & \multicolumn{1}{c}{$K=K_{2} e\left(\frac{E a}{R T}+n_{2} C\right)$} \\
\hline Parámetros & $K_{1}=(37,31 \pm 2,95) * 10^{-6} \mathrm{~Pa} \cdot \mathrm{s}^{\mathrm{n}}$ & $K_{2}=0,01236 \pm 0,0035 \mathrm{~Pa} \cdot \mathrm{s}^{\mathrm{n}}$ \\
de los modelos & $n_{1}=2,6693 \pm 0,165$ & $n_{2}=0,1099 \pm 0,0069$ \\
& $E_{a}=10,97 \pm 1,23 \mathrm{~kJ} / \mathrm{mol}$ & $E_{a}=10,96 \pm 1,30 \mathrm{~kJ} / \mathrm{mol}$ \\
& $r^{2}=0,973$ & $r^{2}=0,970$ \\
\hline
\end{tabular}

\section{Viscosidad aparente}

La Figura 4 muestra que la viscosidad aparente disminuye a medida que aumenta la velocidad de cizalla, a una concentración y temperatura determinadas. Este comportamiento es propio de un fluido no Newtoniano de características seudoplásticas y se debe a la restructuración espacial o alineamiento de las moléculas en el seno de la pulpa por influencia de la cantidad de movimiento impartida por el agitador mecánico. De igual manera, muestra que la viscosidad aparente disminuye a medida que aumenta la temperatura de la pulpa, a una concentración y una velocidad de cizalla determinados. Esta disminución se puede atribuir a la reestructuración espacial molecular en la pulpa provocada por el aumento de la distancia intermolecular debido a la agitación y expansión molecular por el incremento de la temperatura [7]. Una relación lineal de $\ln \left(\eta_{a p p}\right)$ frente al inverso de la temperatura absoluta $(1 / T)$ fue observada e ilustrada en la Figura 5. Este comportamiento se debe a que la viscosidad de un líquido es una función de las fuerzas intermoleculares que restringen el movimiento molecular. Estas fuerzas dependen de la separación intermolecular que determinan el volumen libre y se ven afectadas por el cambio en la temperatura. A temperaturas más altas, la energía térmica de la molécula aumenta con el aumento de las distancias intermoleculares, provocando la reducción de las fuerzas intermoleculares y en consecuencia la viscosidad disminuye [28]. 

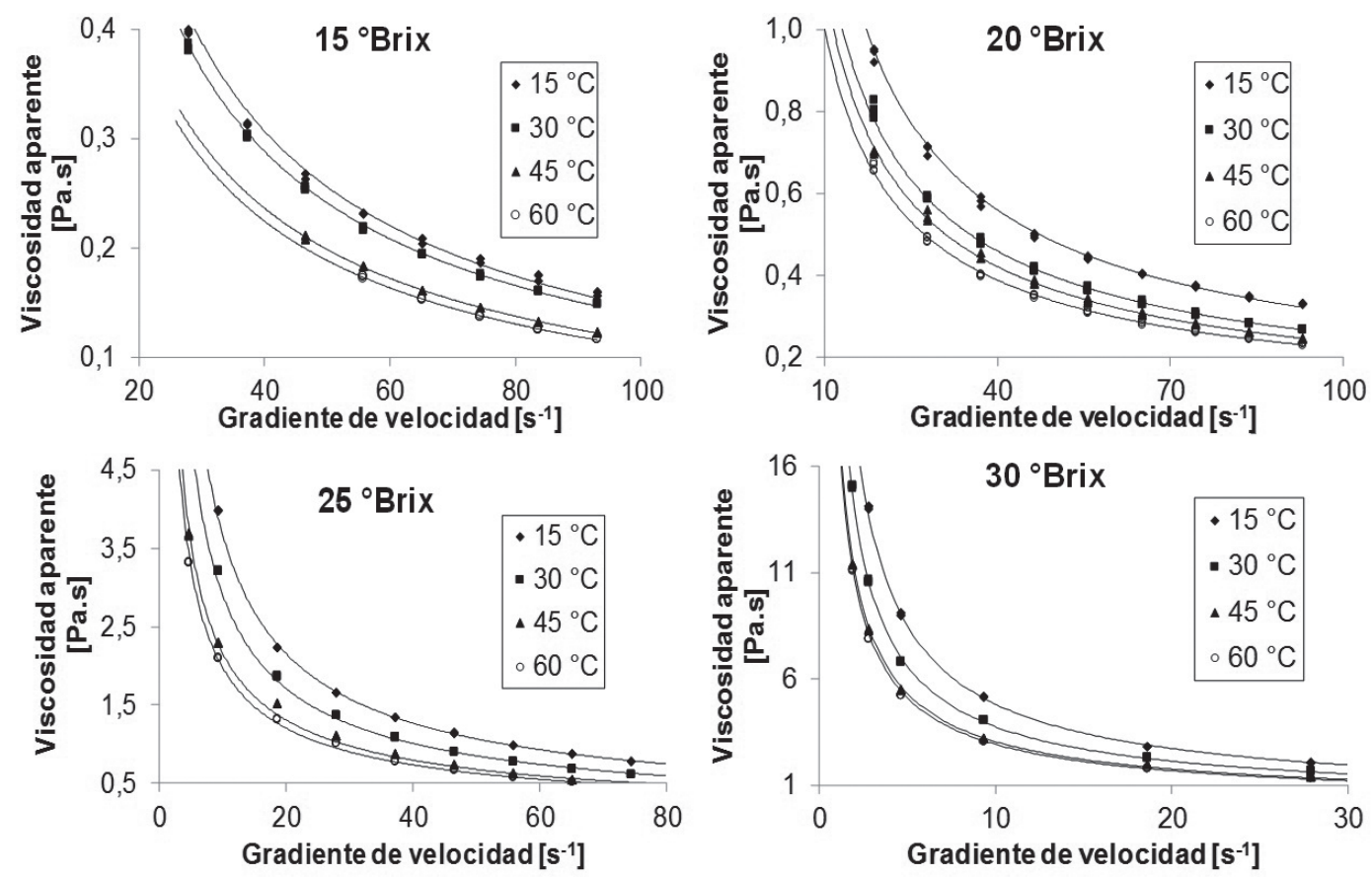

Figura 4. Viscosidad aparente frente a la velocidad de cizalla a diferentes temperaturas de la pulpa.

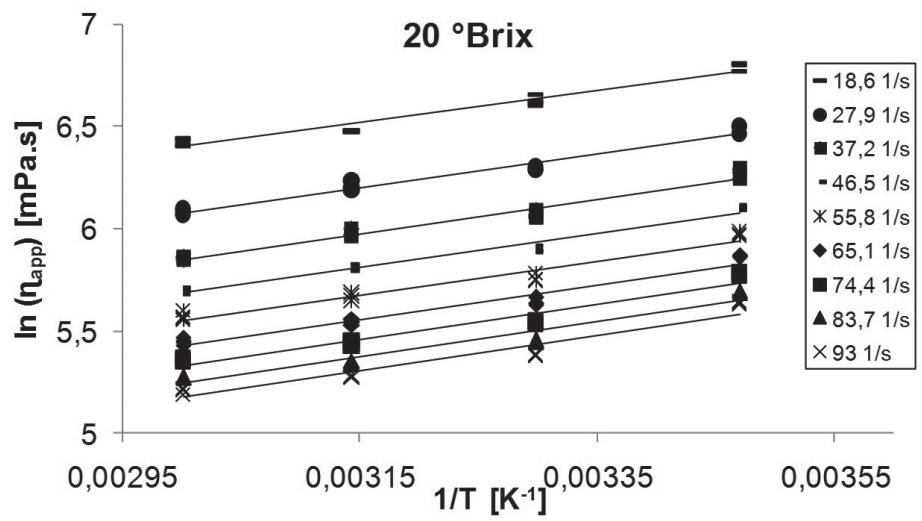

Figura 5. Viscosidad aparente frente al inverso de la temperatura a diferentes gradientes de velocidad y concentración $20^{\circ}$ Brix.

La Tabla 6 muestra los parámetros del modelo de Arrhenius aplicado a la viscosidad aparente. Los resultados indican que el efecto de la temperatura sobre la viscosidad aparente (a una velocidad de cizalla constante) fue más pronunciado a concentraciones más altas. Por otro lado, a una misma concentración, se observa que cuando aumenta la velocidad de cizalla aumenta la energía de activación, cuyos valores se encuentran en el rango $5,39-10,58 \mathrm{~kJ} / \mathrm{mol}$. Estos valores de energía de activación indican la sensibilidad de la viscosidad aparente frente al cambio de la temperatura. Los valores más altos de la energía de activación significan que la viscosidad aparente es relativamente más sensible a los cambios de temperaturas $[7,26,28]$. 
Tabla 6. Efecto de la temperatura sobre la viscosidad aparente de pulpa (Ecuación 2).

\begin{tabular}{|c|c|c|c|c|}
\hline $\begin{array}{c}\text { Velocidad de } \\
\text { cizalla }\left[\mathbf{s}^{-1}\right]\end{array}$ & $\begin{array}{c}\text { Concentración } \\
{\left[{ }^{\circ} B \text { rix }\right]}\end{array}$ & $\eta_{o}[\mathrm{~Pa} . \mathrm{s}]$ & $\begin{array}{c}E a \\
{[\mathrm{~kJ} / \mathrm{mol}]}\end{array}$ & $r^{2}$ \\
\hline 18,6 & $\begin{array}{l}15 \\
20 \\
25 \\
30\end{array}$ & $\begin{array}{c}- \\
0,0514 \pm 0,0073 \\
0,0422 \pm 0,0020 \\
0,0856 \pm 0,0042\end{array}$ & $\begin{array}{c}- \\
6,80 \pm 0,36 \\
9,51 \pm 0,12 \\
8,34 \pm 0,26\end{array}$ & $\begin{array}{c}- \\
0,966 \\
0,998 \\
0,986\end{array}$ \\
\hline 27,9 & $\begin{array}{l}15 \\
20 \\
25 \\
30\end{array}$ & $\begin{array}{c}- \\
0,0338 \pm 0,0048 \\
0,0338 \pm 0,0032 \\
0,0640 \pm 0,0045\end{array}$ & $\begin{array}{c}- \\
7,06 \pm 0,35 \\
9,31 \pm 0,24 \\
8,26 \pm 0,36\end{array}$ & $\begin{array}{c}- \\
0,969 \\
0,991 \\
0,972\end{array}$ \\
\hline 37,2 & $\begin{array}{l}15 \\
20 \\
25 \\
30\end{array}$ & $\begin{array}{c}- \\
0,0252 \pm 0,0038 \\
0,0199 \pm 0,0015 \\
0,0395 \pm 0,0050\end{array}$ & $\begin{array}{c}- \\
7,25 \pm 0,38 \\
10,08 \pm 0,19 \\
8,87 \pm 0,33\end{array}$ & $\begin{array}{l}- \\
0,967 \\
0,995 \\
0,980\end{array}$ \\
\hline 46,5 & $\begin{array}{l}15 \\
20 \\
25 \\
30\end{array}$ & $\begin{array}{l}0,0263 \pm 0,0040 \\
0,0229 \pm 0,0034 \\
0,0175 \pm 0,0023 \\
0,0336 \pm 0,0036\end{array}$ & $\begin{array}{r}5,39 \pm 0,31 \\
7,06 \pm 0,37 \\
9,98 \pm 0,33 \\
8,83 \pm 0,47\end{array}$ & $\begin{array}{l}0,954 \\
0,965 \\
0,984 \\
0,965\end{array}$ \\
\hline 55,8 & $\begin{array}{l}15 \\
20 \\
25 \\
30\end{array}$ & $\begin{array}{l}0,0238 \pm 0,0032 \\
0,0194 \pm 0,0034 \\
0,0135 \pm 0,0021 \\
0,0260 \pm 0,0026\end{array}$ & $\begin{array}{c}5,48 \pm 0,33 \\
7,14 \pm 0,44 \\
10,25 \pm 0,38 \\
9,08 \pm 0,50\end{array}$ & $\begin{array}{l}0,948 \\
0,953 \\
0,980 \\
0,965\end{array}$ \\
\hline 65,1 & $\begin{array}{l}15 \\
20 \\
25 \\
30\end{array}$ & $\begin{array}{l}0,0199 \pm 0,0033 \\
0,0159 \pm 0,0029 \\
0,0118 \pm 0,0021 \\
0,0225 \pm 0,0050\end{array}$ & $\begin{array}{c}5,63 \pm 0,38 \\
7,36 \pm 0,46 \\
10,27 \pm 0,46 \\
9,11 \pm 0,56\end{array}$ & $\begin{array}{l}0,938 \\
0,953 \\
0,972 \\
0,969\end{array}$ \\
\hline 74,4 & $\begin{array}{l}15 \\
20 \\
25 \\
30\end{array}$ & $\begin{array}{l}0,0158 \pm 0,0022 \\
0,0134 \pm 0,0027 \\
0,0106 \pm 0,0020 \\
0,0217 \pm 0,0042\end{array}$ & $\begin{array}{c}5,96 \pm 0,34 \\
7,54 \pm 0,50 \\
10,24 \pm 0,47 \\
8,91 \pm 0,55\end{array}$ & $\begin{array}{l}0,954 \\
0,945 \\
0,972 \\
0,962\end{array}$ \\
\hline 83,7 & $\begin{array}{l}15 \\
20 \\
25 \\
30\end{array}$ & $\begin{array}{l}0,0139 \pm 0,0021 \\
0,0120 \pm 0,0026 \\
0,0103 \pm 0,0019 \\
0,0186 \pm 0,0041\end{array}$ & $\begin{array}{c}6,05 \pm 0,39 \\
7,61 \pm 0,53 \\
10,08 \pm 0,46 \\
9,04 \pm 0,55\end{array}$ & $\begin{array}{l}0,943 \\
0,941 \\
0,971 \\
0,948\end{array}$ \\
\hline 93 & $\begin{array}{l}15 \\
20 \\
25 \\
30\end{array}$ & $\begin{array}{l}0,0141 \pm 0,0023 \\
0,0104 \pm 0,0025 \\
0,0079 \pm 0,0015 \\
0,0137 \pm 0,0030\end{array}$ & $\begin{array}{c}5,83 \pm 0,41 \\
7,79 \pm 0,61 \\
10,58 \pm 0,48 \\
9,64 \pm 0,54\end{array}$ & $\begin{array}{l}0,933 \\
0,927 \\
0,971 \\
0,955\end{array}$ \\
\hline
\end{tabular}

La Tabla 7 muestra que, en el rango de $15-45^{\circ} \mathrm{C}$, el parámetro $\alpha$ de la Ecuación 5 tiende a disminuir cuando la temperatura aumenta a velocidad de cizalla constante, lo cual indica que el efecto de la concentración sobre la viscosidad aparente es ligeramente más alto a temperaturas bajas.

Para un valor de la velocidad de cizalla, los efectos combinados de la temperatura y la concentración sobre la viscosidad aparente pueden ser expresados por la Ecuación 8. Los parámetros de esta expresión se muestran en la Tabla 8 y se observa que los valores de la energía de activación son muy similares, mientras que los valores de $\varepsilon$ tienen tendencia a disminuir cuando aumenta la velocidad de cizalla.

Los efectos combinados de la temperatura, concentración y velocidad de cizalla sobre la viscosidad aparente se expresan mediante la Ecuación 9, la cual es mostrada con sus parámetros ( \pm desviación) en la Ecuación 13.

$$
\eta_{\text {app }}=(0,02132 \pm 0,0014) \dot{\gamma}^{-0,7754} e^{\left[\left(\frac{1299,48 \pm 16,08}{T}\right)+(0,0923 \pm 0,0011) C\right] ; \quad\left(r^{2}=0,993\right)}
$$

Donde $\eta_{a p p}:$ Pa.s; $\dot{\gamma}: \mathrm{s}^{-1} ; C:{ }^{\circ}$ Brix y $T: \mathrm{K}$. 
Tabla 7. Efecto de la concentración sobre la viscosidad aparente de pulpa (Ecuación 5).

\begin{tabular}{|c|c|c|c|c|}
\hline $\begin{array}{l}\text { Velocidad de } \\
\text { cizalla }\left[\mathbf{s}^{-1}\right]\end{array}$ & $\begin{array}{c}\text { Temperatura } \\
{\left[{ }^{\circ} \mathrm{C}\right]}\end{array}$ & $\begin{array}{l}\eta_{1} \cdot 10^{6} \\
{[\mathrm{~Pa} . \mathrm{s}]}\end{array}$ & $\alpha$ & $r^{2}$ \\
\hline 18,6 & $\begin{array}{l}15 \\
30 \\
45 \\
60\end{array}$ & $\begin{array}{c}1202,98 \pm 243,1 \\
1282,24 \pm 78,23 \\
982,54 \pm 77,85 \\
605,79 \pm 32,66\end{array}$ & $\begin{array}{l}2,294 \pm 0,192 \\
2,214 \pm 0,179 \\
2,245 \pm 0,231 \\
2,358 \pm 0,157\end{array}$ & $\begin{array}{l}0,952 \\
0,940 \\
0,928 \\
0,964\end{array}$ \\
\hline 27,9 & $\begin{array}{l}15 \\
30 \\
45 \\
60\end{array}$ & $\begin{array}{l}911,92 \pm 60,2 \\
919,27 \pm 60,0 \\
1032,3 \pm 71,0 \\
464,72 \pm 34,1\end{array}$ & $\begin{array}{l}2,282 \pm 0,193 \\
2,217 \pm 0,192 \\
2,130 \pm 0,227 \\
2,347 \pm 0,202\end{array}$ & $\begin{array}{l}0,945 \\
0,943 \\
0,945 \\
0,943\end{array}$ \\
\hline 37,2 & $\begin{array}{l}15 \\
30 \\
45 \\
60\end{array}$ & $\begin{array}{l}780,44 \pm 89,8 \\
791,87 \pm 51,9 \\
946,44 \pm 73,1 \\
439,95 \pm 29,0\end{array}$ & $\begin{array}{l}2,262 \pm 0,202 \\
2,191 \pm 0,192 \\
2,085 \pm 0,225 \\
2,286 \pm 0,192\end{array}$ & $\begin{array}{l}0,940 \\
0,943 \\
0,949 \\
0,945\end{array}$ \\
\hline 46,5 & $\begin{array}{l}15 \\
30 \\
45 \\
60\end{array}$ & $\begin{array}{c}694,50 \pm 49,4 \\
728,08 \pm 47,09 \\
735,39 \pm 36,59 \\
421,39 \pm 29,87\end{array}$ & $\begin{array}{l}2,246 \pm 0,208 \\
2,159 \pm 0,190 \\
2,106 \pm 0,146 \\
2,252 \pm 0,207\end{array}$ & $\begin{array}{l}0,955 \\
0,953 \\
0,960 \\
0,944\end{array}$ \\
\hline 55,8 & $\begin{array}{l}15 \\
30 \\
45 \\
60\end{array}$ & $\begin{array}{l}652,89 \pm 46,1 \\
657,72 \pm 42,3 \\
716,28 \pm 34,5 \\
463,62 \pm 21,3\end{array}$ & $\begin{array}{l}2,220 \pm 0,207 \\
2,143 \pm 0,189 \\
2,064 \pm 0,141 \\
2,176 \pm 0,135\end{array}$ & $\begin{array}{l}0,953 \\
0,952 \\
0,961 \\
0,963\end{array}$ \\
\hline 65,1 & $\begin{array}{l}15 \\
30 \\
45 \\
60\end{array}$ & $\begin{array}{l}627,27 \pm 43,7 \\
640,00 \pm 40,0 \\
669,83 \pm 31,6 \\
431,64 \pm 20,1\end{array}$ & $\begin{array}{l}2,194 \pm 0,204 \\
2,111 \pm 0,184 \\
2,043 \pm 0,139 \\
2,160 \pm 0,137\end{array}$ & $\begin{array}{l}0,954 \\
0,953 \\
0,961 \\
0,961\end{array}$ \\
\hline 74,4 & $\begin{array}{l}15 \\
30 \\
45 \\
60\end{array}$ & $\begin{array}{l}640,73 \pm 42,74 \\
604,37 \pm 37,72 \\
637,85 \pm 29,97 \\
414,02 \pm 19,17\end{array}$ & $\begin{array}{l}2,151 \pm 0,195 \\
2,095 \pm 0,184 \\
2,023 \pm 0,138 \\
2,141 \pm 0,136\end{array}$ & $\begin{array}{l}0,957 \\
0,952 \\
0,960 \\
0,961\end{array}$ \\
\hline 83,7 & $\begin{array}{l}15 \\
30 \\
45 \\
60\end{array}$ & $\begin{array}{c}615,16 \pm 40,1 \\
585,14 \pm 36,2 \\
567,93 \pm 27,9 \\
412,38 \pm 18,5\end{array}$ & $\begin{array}{l}2,134 \pm 0,191 \\
2,076 \pm 0,182 \\
2,028 \pm 0,145 \\
2,111 \pm 0,132\end{array}$ & $\begin{array}{l}0,947 \\
0,958 \\
0,957 \\
0,962\end{array}$ \\
\hline 93 & $\begin{array}{l}15 \\
30 \\
45 \\
60\end{array}$ & $\begin{array}{l}549,32 \pm 36,4 \\
519,40 \pm 33,0 \\
595,33 \pm 27,0 \\
412,09 \pm 18,2\end{array}$ & $\begin{array}{l}2,151 \pm 0,194 \\
2,093 \pm 0,187 \\
1,988 \pm 0,133 \\
2,086 \pm 0,130\end{array}$ & $\begin{array}{l}0,966 \\
0,965 \\
0,961 \\
0,963\end{array}$ \\
\hline
\end{tabular}

Los datos experimentales de la viscosidad aparente distribuidos aleatoriamente sobre una línea recta de mostraron un alto grado de ajuste al modelo $45^{\circ}$ en el gráfico de viscosidad aparente predicha propuesto (Ecuación 13), debido a que están versus viscosidad aparente experimental (Figura 6).

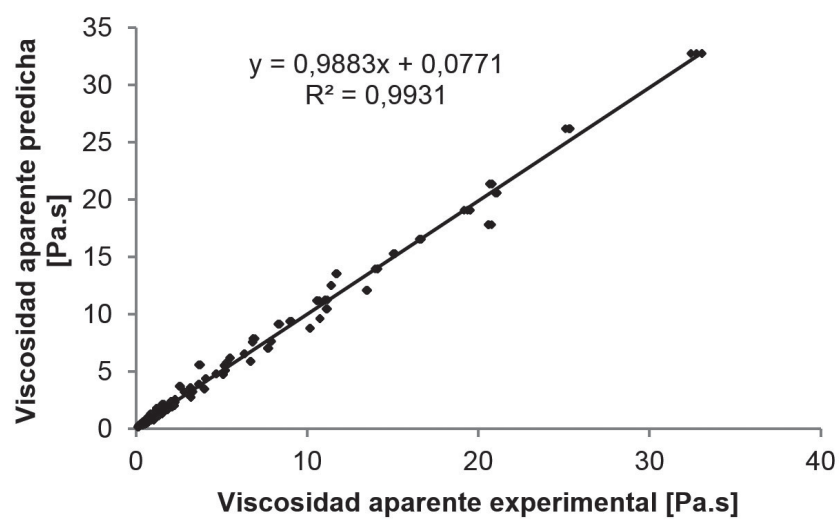

Figura 6. Curva de viscosidad aparente predicha frente a la experimental. 
Tabla 8. Efecto combinado de temperatura $(T)$ y concentración $(C)$ sobre la viscosidad aparente de la pulpa. Ecuación 8.

\begin{tabular}{|c|c|c|c|c|}
\hline $\begin{array}{l}\text { Velocidad de } \\
\left.\text { cizalla [ } \mathbf{s}^{-1}\right]\end{array}$ & $\begin{array}{l}\delta .10^{6} \\
{[\mathrm{~Pa} . \mathrm{s}]}\end{array}$ & $\varepsilon$ & $\begin{array}{c}E a \\
{[\mathrm{~kJ} / \mathrm{mol}]}\end{array}$ & $r^{2}$ \\
\hline 0,93 & $59,31 \pm 2,93$ & $2,634 \pm 0,124$ & $10,19 \pm 0,52$ & 0,966 \\
\hline 1,86 & $69,42 \pm 3,27$ & $2,196 \pm 0,118$ & $12,17 \pm 0,54$ & 0,966 \\
\hline 2,79 & $22,36 \pm 1,25$ & $2,463 \pm 0,129$ & $11,94 \pm 0,71$ & 0,945 \\
\hline 4,65 & $33,35 \pm 1,87$ & $2,311 \pm 0,128$ & $11,15 \pm 0,71$ & 0,947 \\
\hline 9,3 & $24,42 \pm 1,31$ & $2,319 \pm 0,112$ & $10,53 \pm 0,70$ & 0,946 \\
\hline 18,6 & $35,05 \pm 1,48$ & $2,269 \pm 0,089$ & $8,65 \pm 0,69$ & 0,958 \\
\hline 27,9 & $28,51 \pm 1,31$ & $2,250 \pm 0,096$ & $8,53 \pm 0,75$ & 0,949 \\
\hline 37,2 & $19,49 \pm 0,91$ & $2,220 \pm 0,097$ & $9,14 \pm 0,77$ & 0,965 \\
\hline 46,5 & $18,00 \pm 0,82$ & $2,199 \pm 0,093$ & $9,08 \pm 0,77$ & 0,968 \\
\hline 55,8 & $16,00 \pm 0,68$ & $2,165 \pm 0,087$ & $9,28 \pm 0,75$ & 0,943 \\
\hline 65,1 & $15,02 \pm 0,64$ & $2,139 \pm 0,087$ & $9,32 \pm 0,75$ & 0,948 \\
\hline 74,4 & $15,81 \pm 0,66$ & $2,111 \pm 0,085$ & $9,14 \pm 0,74$ & 0,942 \\
\hline 83,7 & $14,69 \pm 0,60$ & $2,091 \pm 0,084$ & $9,24 \pm 0,73$ & 0,943 \\
\hline 93 & $11,04 \pm 0,46$ & $2,096 \pm 0,085$ & $9,75 \pm 0,74$ & 0,945 \\
\hline
\end{tabular}

\section{Comportamiento reológico de la pulpa de} mango por Curva Maestra

La técnica de curva maestra fue usada para modelar el comportamiento reológico general de la pulpa de mango concentrada. Debido a la tendencia similar que tienen las curvas de esfuerzo de corte frente a la velocidad de cizalla de la pulpa a diferentes temperaturas y concentraciones, las curvas de esfuerzo de corte a 15,45 y $60^{\circ} \mathrm{C}$ fueron desplazadas horizontalmente a lo largo del eje de velocidad de cizalla a una temperatura de referencia de $30^{\circ} \mathrm{C}$ y un esfuerzo de corte base de 10Pa.

El factor de corrección de temperatura utilizado para obtener las curvas maestras a diferentes temperaturas es presentado en la Tabla 9 para cada concentración. La Figura 7 muestra los resultados de las curvas maestras en escala logarítmica para cada concentración y la línea recta obtenida fue atribuida a la superposición de la curva por el desplazamiento horizontal de los datos. Las curvas maestras de cada concentración fueron ajustadas a la Ecuación 11 con altos valores de $r^{2}$ y los parámetros son presentados en la Tabla 10. El índice de consistencia se incrementa y el índice de comportamiento de flujo disminuye con la concentración, esto implica que cuando la concentración aumenta, la viscosidad y la seudoplasticidad de las pulpas aumentan, resultados similares fueron obtenidos en investigaciones de jugos concentrados de cítricos [29] y jugos concentrados de guanábana [27].

Tabla 9. Factores de corrección de temperatura y concentración $\left(a_{T}\right.$ y $\left.a_{C}\right)$.

\begin{tabular}{cccccc}
\hline \multirow{2}{*}{$\begin{array}{c}\text { Concentración } \\
{\left[{ }^{\circ} \text { Brix] }\right.}\end{array}$} & \multicolumn{5}{c}{$\boldsymbol{a}_{T}$} \\
\cline { 2 - 5 } & $\mathbf{1 5}$ & $\mathbf{3 0}$ & $\mathbf{4 5}$ & $\mathbf{6 0}$ & \\
\hline 15 & 0,670 & 1 & 2,654 & 3,379 & 3,3445 \\
20 & 0,450 & 1 & 1,465 & 2,254 & 1 \\
25 & 0,343 & 1 & 5,783 & 8,314 & 0,005682 \\
30 & 0,194 & 1 & 4,834 & 5,424 & 0,000316 \\
\hline
\end{tabular}


Tabla 10. Parámetros de la Ecuación 11 a diferentes concentraciones.

\begin{tabular}{cccc}
\hline $\begin{array}{c}\text { Concentración } \\
\text { ['Brix] }\end{array}$ & $\begin{array}{c}\boldsymbol{K}^{\prime} \\
{\left[\mathrm{Pa} \cdot \mathbf{s}^{\mathrm{n}}\right]}\end{array}$ & $\boldsymbol{n}$, & $\boldsymbol{r}^{\mathbf{2}}$ \\
15 & $5,7198 \pm 0,061$ & $0,1887 \pm 0,003$ & 0,975 \\
20 & $6,3827 \pm 0,057$ & $0,2561 \pm 0,002$ & 0,990 \\
25 & $19,807 \pm 0,219$ & $0,2000 \pm 0,003$ & 0,964 \\
30 & $27,070 \pm 0,191$ & $0,1579 \pm 0,002$ & 0,977 \\
\hline
\end{tabular}

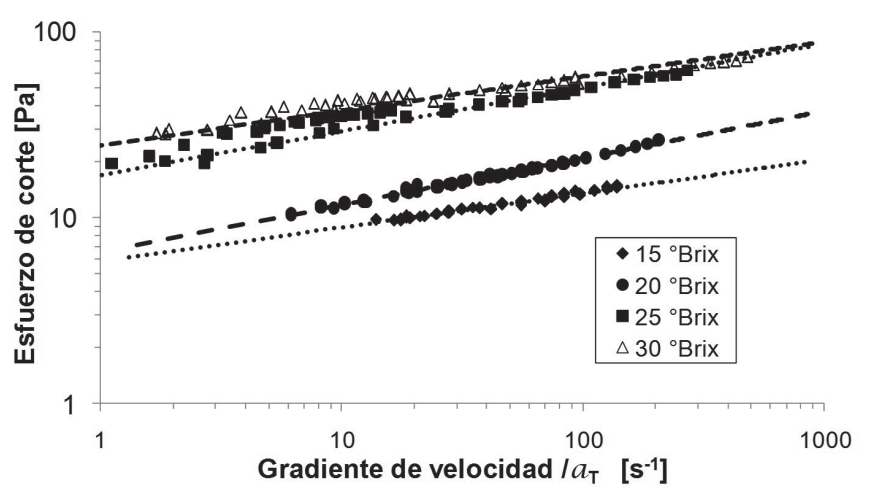

Figura 7. Curvas maestras de esfuerzo de corte frente a la primera velocidad de cizalla reducida.

Las curvas maestras de las cuatro concentraciones $\left(15,20,25\right.$ y $30^{\circ}$ Brix) fueron de nuevo desplazadas horizontalmente a lo largo del eje la velocidad de cizalla a una concentración de referencia de $20^{\circ}$ Brix y un esfuerzo de corte base de 10Pa usando el factor de desplazamiento de concentración,, mostrados en la Tabla 9. Fue obtenida una única curva maestra (Figura 8 ) que describe el comportamiento final de la pulpa de mango como una función del segundo gradiente reducido. Esta curva maestra final fue ajustada a la Ecuación 12 y se muestra con los parámetros ( \pm desviación) en la Ecuación 14.

Finalmente, se encontró una relación entre el factor de desplazamiento de concentración y la concentración representada por la Ecuación 15.

$$
\begin{gathered}
\sigma=(9,9498 \pm 0,182)\left(\frac{\dot{\gamma}}{a_{C} a_{T}}\right)^{(0,1401 \pm 0,002)} ; \quad\left(r^{2}=0,916\right) \\
a_{C}=191,596 e-0,2695 C ; \quad\left(r^{2}=0,991\right)
\end{gathered}
$$

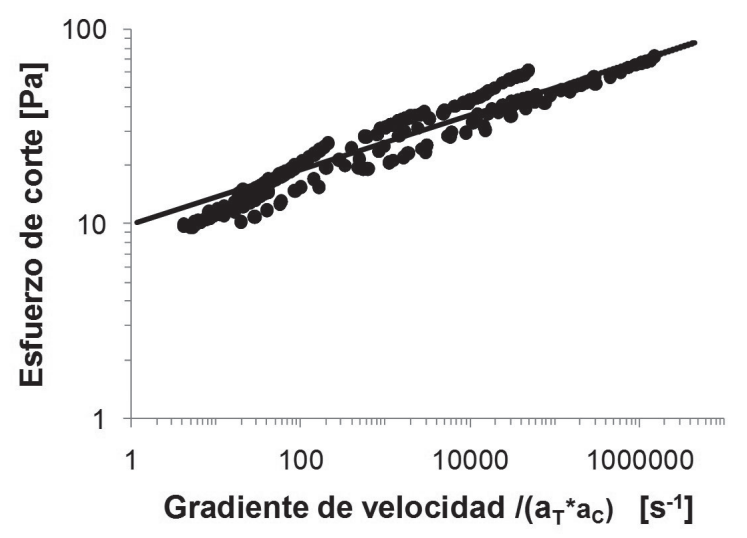

Figura 8. Curva maestra de esfuerzo de corte frente a la segunda velocidad de cizalla reducida. 
Los resultados anteriores implican que el comportamiento reológico de la pulpa concentrada de mango puede ser descrita por curvas de desplazamiento basadas en la técnica de curva maestra, la cual es también conocida como técnica de superposición gradiente-temperaturaconcentración. Estas curvas maestras son recomendadas para comparar los datos de diferentes pulpas y jugos de frutas $[9,30]$ y en particular, la técnica de doble desplazamiento produce una única representación de datos reológicos cubriendo un amplio rango de temperatura y concentración.

\section{Conclusiones}

De acuerdo con los resultados obtenidos en esta investigación, se establece que la pulpa de mango de la variedad Tommy Atkins se comporta como un fluido no Newtoniano seudoplástico sin tixotropía y los datos experimentales fueron ajustados adecuadamente por el modelo de Ostwald de Waele.

El índice de consistencia mostró correlación positiva con el inverso de la temperatura absoluta y también incrementó con el aumento de la concentración. Por otro lado, la viscosidad aparente disminuyó con el incremento de la temperatura y aumentó con el incremento de la concentración.

Para predecir el índice de consistencia y la viscosidad aparente en función de la temperatura, concentración y el efecto combinado de ambas, los modelos matemáticos utilizados presentaron un buen ajuste de los datos $\left(r^{2}>0,92\right)$.

La ley de Potencia presentó un buen ajuste de los datos de la única curva maestra obtenida para describir el comportamiento de flujo de la pulpa de mango como una función de la velocidad de cizalla corregida por el desplazamiento de la temperatura y por desplazamiento de la concentración.

\section{Agradecimientos}

Los autores extienden su agradecimiento al laboratorio de Ingeniería Aplicada de la Universidad de Córdoba (Colombia) por el apoyo financiero del presente trabajo.

\section{Referencias bibliográficas}

[1] Ibarz A, Gonzalez C, Esplugas S. Rheology of clarified passion fruit juices. Fruit Process. 1996;6(8):330-3.
[2] Holdsworth SD. Applicability of rheological models to the interpretation of flow and processing behaviour of fluid food products. $J$ Texture Stud. 1971;2:393-418.

[3] Vitali AA, Rao MA. Flow properties of lowpulp concentrated orange juice: Effect of temperature and concentration. J Food Sci.1984;49:882-8.

[4] Hernandez E, Chen CS, Johnson J, Carter RD. Viscosity changes in orange juice after ultrafiltration and evaporation. J Food Eng. 1995;25(3):387-96.

[5] Ahmed J, Shivhare U, Raghavan G. Rheological characteristics and kinetics of color degradation of green chilli puree. J Food Eng. 2000;44(4):239-44.

[6] Ahmed J, Shivhare U, Singh, P. Colour kinetics and rheology of coriander leaf puree and storage characteristics of the paste. Food Chem. 2004;84:604-11.

[7] Haminiuk C, Sierakowski M, Vidal J, Masson M. Influence of temperature on the rheological of whole araçá pulp (Psidium cattleianum sabine). Lebensm Wiss Technol. 2006;39:42630.

[8] Pelegrine D, Silva F, Gasparetto C. Rheological Behvior of Pineapple and Mango Pulps. Lebensm Wiss Technol. 2002;35:645-8.

[9] Steffe JF. Rheological methods in food process engineering. 2 ed. Estados Unidos: Freeman Press; 1996.

[10]Dak M, Verma R, Sharma G. Flow characteristics of juice of "Totapuri" mangoes. J Food Eng. 2006;76:557-61.

[11] Branco IG, Gasparetto C. A response surface methodology applied to the study of temperature effect on the rheological behavior of ternaries mixtures with mango pulp and orange and carrot juices. Ciênc Tec Alim. 2003;23:166-71.

[12] Malkin A, Isayev A. Rheology: Concepts, Methods, and Applications. 2 ed. Canadá: ChemTec Publishing; 2012.

[13] Mackey KL, Ofoli RY, Morgan RG, Steffe JF. Rheological modeling of potato flour during extrusion cooking. J. Food Proc. Eng.1989;12:1-11.

[14]Dak M, Verma R, Jaaffrey S. Effect of temperature and concentration on Rheological properties of "Kesar" mango juice. J Food Eng. 2007;80:1011-5.

[15]A.O.A.C. Official Methods of Analysis of the Association of Official Analytical Chemist. 
Estados Unidos: Editorial Association of Official Analytical Chemists; 1990.

[16] Ramos AM, Ibarz A. Thixotropy of orange concentrate and quince puree. J Texture Stud.1998;29(3):313-24.

[17] Sharma S, Le Maguer M, Liptay A, Poysa V. Effect of composition on the reological properties of tomato thin pulp. Food Res Int.. 1996;29(2):175-9.

[18] Krokida MK, Maroulis ZB, Saravacos GD. Rheological properties of fluid fruit and vegetable puree products: compilation of literature data. Int $\mathrm{J}$ Food Properties. 2001;4(2):179-200.

[19] Khandari P, Gill BS, Sodhi NS. Effect of concentration and temperature on the Rheology of mango pulp. J. Food Sci. Technol. 2002;39(2):152-4.

[20] Rao MA, Bourne MC, Cooley HJ. Flow properties of tomato concentrates. J Texture Stud. 1981;12:521-38.

[21]Dak M, Charan R, Kumar M. Mathematical models for prediction of rheological parameters of pineapple juice. Int J Food Eng. 2008;4(3):1-16.

[22]Dak M, Charan R, Jaaffrey SN. Rheological properties of tomato concentrate. Int J Food Eng. 2008;4(7):1-17.

[23] Vitali AA, Rao M.A. Flow properties of low- pulp concentrated orange juice: effect of temperature and concentration. J. Food Sci. 1984;49;882-8.

[24] Kaya A, Belibagli K. Rheology of solid Gaziantep Pekmez. J Food Eng. 2002;54:221-6.

[25] Juszczak L, Fortuna T. Effect of temperature and soluble solid content on the viscosity of cherry juice concentrate. Int Agrophysics. 2004;18:17-21.

[26]Kaya A, Sözer N. Rheological behavior of sour pomegranate juice concentrates (Punica granatum L.). Int J Food Sci Tech. 2005;40:223-7.

[27]Quek M, Chin N, Yusof Y. Modelling of rheological behaviour of soursop juice concentrates using shear rate-temperatureconcentration superposition. J Food Eng. 2013;118(4):380-5.

[28] Toğrul H, Arslan N. Mathematical model for prediction of apparent viscosity of molasses. J Food Eng. 2004;62:281-9.

[29]Chin NL, Chan SM, Yusof YA, Chuah TG, Talib RA. Modelling of rheological behaviour of pummelo juice concentrates using mastercurve. J Food Eng. 2009;93(2):134-40.

[30]Chuah T, Ling H, Chin N, Choong T, Razi F. Effects of temperature on Rheological Behavior of Drgaon Fruit (Hylocereus sp.) Juice. Int J Food Eng. 2008;4(7):1-28. 